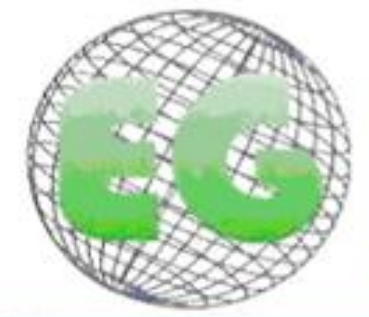

$\mathrm{N}^{\circ} 37$

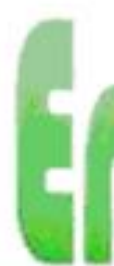

ISSN 1695-6141
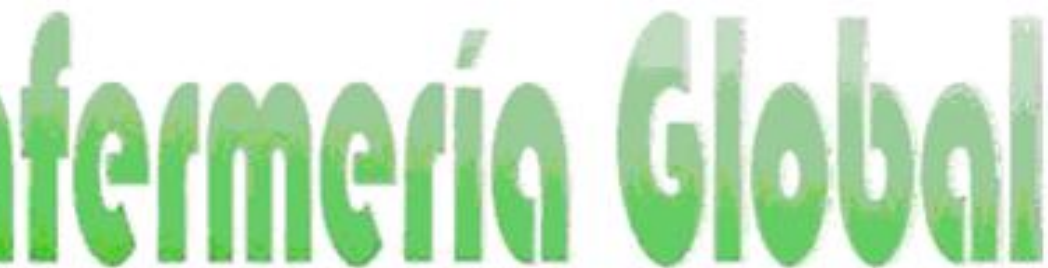

Revista electrónica trimestral de Enfermeria

Enero 2015

www.um.es/egloball

\title{
CLÍNICA
}

\section{Cuidados de los pies: el conocimiento de las personas con diabetes mellitus inscritos en el programa de salud familiar}

Cuidados com os pés: o conhecimento de indivíduos com diabetes mellitus cadastrados no programa saúde da familia

Care of the feet: the knowledge of individuals with diabetes mellitus registered family health program

\section{"Silva, Pollyane Liliane ${ }^{* *}$ Rezende, Marina Pereira ${ }^{* *}$ Ferreira, Lúcia Aparecida ***Dias, Flavia Aparecida *Helmo, Fernanda Rodrigues ****Silveira, Fabíola Cristina Oliveira}

*Enfermera. Especialista con énfasis en Salud del Adulto por la Universidad Federal del Triángulo Minero (UFTM). E-mail: pollyane.s@hotmail.com "**Doctora en Enfermería. Profesora del Departamento de Enfermería en Educación y Salud Comunitaria de la UFTM. ***Enfermera. Máster en Atención a la Salud. Profesora Sustituta del Departamento de Enfermería en Educación y Salud Comunitaria de la UFTM. ****Enfermera Graduada por la UFTM.Brasil.

\author{
Palabras Clave: Diabetes Mellitus; Conocimiento; Pie Diabético \\ Palavras-chave: Diabetes Mellitus; Conhecimento; Pé Diabético \\ Keywords: Diabetes Mellitus; Knowledge; Diabetic Foot
}

\section{RESUMEN}

El objetivo es identificar el conocimiento de los individuos con Diabetes mellitus tipo 1 o 2 catastrados en el Programa HIPERDIA, correlacionar el tiempo de estudio con el número de respuestas concordantes acerca de los cuidados con los pies y describir las complicaciones según tiempo de diagnóstico.

Estudio analítico, exploratorio y transversal, con análisis cuantitativo, realizado con 116 individuos diabéticos tipo 1 o 2 acompañados en el Programa HIPERDIA en una Unidad Matricial de Salud. Se evidenció que la mayor parte de los individuos era del sexo femenino, edad promedia de 49,9 años, enseñanza básica incompleta, posee diabetes tipo 2. 69\% presentaban hipertensión arterial y 45,7\% dislipidemia. La mayoría refirió conductas adecuadas para el cuidado con los pies, $98,3 \%$ hallaban importante cuidar de los pies para prevenir el pie diabético, pero, 19\% realizaba el examen de los pies con profesional de salud por lo menos una vez al año. Los tests estadísticos muestrearon que hubo una baja correlación entre el tiempo de estudio y el número de respuestas concordantes acerca de los cuidados con los pies, pero no fue significativa $(r s=0,026 ; p=0,781)$. De entre aquellos con hasta 10 
años de diagnóstico, $79,7 \%$ no presentaron complicaciones y $62,2 \%$ mencionaron tener, para aquellos con 10 años o más $\left(x^{2}=19,83 ; p<0,001\right)$.

Se concluyó que tener ciencia del nivel de conocimiento del individuo diabético sobre los cuidados con los pies permite al profesional de salud direccionar las acciones de educación en salud que promuevan e incentiven esta práctica.

\section{RESUMO}

O objetivo é identificar o conhecimento dos indivíduos com Diabetes Mellitus tipo 1 ou 2 cadastrados no Programa HIPERDIA, correlacionar o tempo de estudo com o número de respostas concordantes acerca dos cuidados com os pés e descrever as complicações segundo tempo de diagnóstico.

Estudo analítico, exploratório e transversal, com análise quantitativa, realizado com 116 indivíduos diabéticos tipo 1 ou 2 acompanhados no Programa HIPERDIA em uma Unidade Matricial de Saúde. Evidenciou-se que a maior parte dos indivíduos era do sexo feminino, idade média de 49,9 anos, ensino fundamental incompleto, possui diabetes tipo 2. 69\% apresentavam hipertensão arterial e 45,7\% dislipidemia. A maioria referiu condutas adequadas para o cuidado com os pés, $98,3 \%$ achavam importante cuidar dos pés para prevenir o pé diabético, e 19\% realizavam o exame dos pés com profissional de saúde pelo menos uma vez ao ano. Houve uma correlação baixa entre o tempo de estudo e o número de respostas concordantes acerca dos cuidados com os pés, mas não foi significativa $(r s=0,026 ; p=0,781)$. Dentre aqueles com até 10 anos de diagnóstico, $79,7 \%$ não apresentaram complicações e $62,2 \%$ mencionaram ter, para aqueles com 10 anos ou mais $\left(X^{2}=19,83\right.$; $p<0,001)$.

Conclui-se que ter ciência do nível de conhecimento do indivíduo diabético sobre os cuidados com os pés permite ao profissional de saúde direcionar as ações de educação em saúde que visem promover e incentivar esta prática.

\section{ABSTRACT}

The Objective is to identify the knowledge of individuals with diabetes mellitus type 1 or 2 registered in the HIPERDIA program, correlate study time with the number of concordant answers about foot care and describe the complications on second time of diagnosis.

Analytical study, exploratory, cross-sectional quantitative analysis, conducted with 116 individuals with diabetes type 1 or 2 followed at HIPERDIA Program in a Health Unit Matrix became evident that most of the individuals were female, mean age 49, 9 years, elementary school, has type 2 diabetes. $69 \%$ had hypertension and $45.7 \%$ dyslipidemia. The majority reported appropriate behaviors in their day-to-day for foot care, $98.3 \%$ thought important to take care of your feet to prevent diabetic foot, however, $19 \%$ performed the examination of the feet to a health professional at least once a year. Statistical tests showed that there was low correlation between study time and the number of concordant answers about foot care, but was not significant $(r s=0.026, p=0.781)$. Among those up to 10 years of diagnosis, $79.7 \%$ had no complications and $62.2 \%$ said they were for those aged 10 or more $\left(x^{2}=19.83, p<0.001\right)$.

We conclude that having knowledge of the individual's level of knowledge about diabetic foot care allows the health professional to direct the actions of health education aimed at promoting and encouraging this practice.

\section{INTRODUCCIÓN}

La Diabetes Mellitus (DM) es denominada por un síndrome derivado de problemas metabólicos, siendo la hiperglicemia el factor de agravamiento. Los desajustes de los niveles glicémicos son debido a defectos de la acción o de la secreción de la insulina 0 de ambos ${ }^{(1)}$.

En Brasil el 11\% de la población con más de 40 años es portadora de diabetes tipo 2, lo que representa cerca de 5 millones de personas en todo el país. El sudeste demuestra ser la región brasileña que presenta más adultos con edad igual o superior 
a 18 años con diagnóstico médico referido para diabetes, siendo $5,8 \%$ de su población, destacando la baja escolaridad con $7,5 \%$ y el sexo femenino con $6,1 \%{ }^{(2)}$.

El aumento de la urbanización, de la expectativa de vida y de los malos hábitos como el sedentarismo, la dieta inadecuada y la obesidad influyen directamente en la incidencia y prevalencia de DM en el mundo, caracterizándola como epidemia mundial ${ }^{(3)}$.

La Organización Mundial de Salud (OMS) y la Asociación Americana de Diabetes (ADA) clasifican la DM en tipo 1, tipo 2, gestacional y otros tipos específicos (Maturity Onset Diabetes of the Young - MODY, Latent Autoimmune Diabetes of the Adult LADA) ${ }^{(1)}$.

La DM tipo 1 resulta de la destrucción de las células beta pancreáticas debido a una reacción auto-inmune también denominada como forma idiopática. Esa reacción es mucho más rápida en niños, ataca también a los adolescentes y es lentamente progresiva en los adultos. La DM tipo 2 es responsable del 90 a $95 \%$ de los casos de diabetes, siendo resultante de la resistencia a la acción de la insulina y de la disfunción de las células beta. Puede alcanzar a individuos de cualquier franja de edad, siendo más frecuente en aquellos con más de 40 años ${ }^{(1)}$.

Se estima que en 2030, 300 millones de individuos sufran de diabetes ${ }^{(1)}$. La DM es responsable del $9 \%$ de la mortalidad mundial, ejerce gran impacto en la expectativa y en la calidad de vida, reducción de la productividad en el trabajo y jubilación precoz. Es también la primera causa de hospitalizaciones y amputaciones no traumáticas de miembros inferiores ${ }^{(3)}$.

Las complicaciones de la DM se componen en macrovasculares (enfermedad arterial coronaria, enfermedad cerebrovascular y vascular periférica) y las microvasculares (retinopatía, nefropatía y neuropatía). Estas complicaciones poseen alto índice de morbo-mortalidad especialmente asociadas a otros factores como hipertensión arterial, tabaquismo y dislipidemias ${ }^{(3)}$.

Entre las complicaciones microvasculares destaca el pie diabético, definido como estado de infección, ulceración o destrucción de las estructuras profundas de los pies, acompañada de las anormalidades neurológicas y diversos grados de enfermedad vascular periférica, en los miembros inferiores de pacientes con DM ${ }^{(4)}$.

Las ulceraciones en los pies son desencadenadas por hábitos inadecuados como andar descalzo, uso de zapatos apretados, corte inadecuado de las uñas, así como, presencia de callos y rajaduras en los pies. La progresión del pie diabético culmina no solamente en pérdidas físicas, sino también en psicológicas, pues afecta directamente a la autoestima ${ }^{(5)}$.

Estimativas apuntan que aproximadamente $15 \%$ de los individuos que presentan DM van a desarrollar alguna lesión en los pies a lo largo de la vida, y que solo $19 \%$ de los individuos ya diagnosticados y admitidos en servicios de atención terciaria realizaron el examen de los pies después de quitar medias y calzados ${ }^{(6)}$.

Ante esto, se nota que la adopción de medidas simples en el cuidado con los pies y el control eficaz de la DM contribuye a la prevención de esta complicación ${ }^{(5,7)}$. 
El conocimiento del individuo diabético acerca del cuidado con los pies se refleja en la reducción del riesgo de desarrollo de úlceras, mejora de la calidad de vida, además de disminuir gastos con ingresos y amputaciones ${ }^{(8)}$, por eso es importante conocer sus experiencias previas en relación a este tema, permitiendo al profesional de salud incentivar y estimular una postura pro-activa y copartícipe en el autocuidado. Para que el individuo adquiera su responsabilidad en la terapéutica, es necesario que domine conocimientos y desarrolle habilidades para el autocuidado. En la prevención de complicaciones en los miembros inferiores es fundamental la presencia tanto del conocimiento como del comportamiento para el cuidado con los pies ${ }^{(6)}$.

Retomando el principio fundamental de que el Sistema Único de Salud (SUS) debe responder a las necesidades de la población y para que la referencia sea efectiva, es necesario que el enfermero, en la condición de educador y de profesional, viabilice el encaminamiento de individuos e identifique el conocimiento de los diabéticos acompañados en la Atención Primaria a la Salud. De esa forma, identificar el conocimiento de los individuos que presentan DM acerca de los cuidados con los pies permitirá a las instituciones públicas de salud desarrollar y dirigir acciones educativas para la prevención de esta complicación. Se observa en la práctica que los individuos adultos con DM que poseen baja escolaridad presentan mayor dificultad para la comprensión, el desarrollo adecuado del cuidado con los pies y la identificación de las complicaciones, lo que nos incita a realizar ese estudio.

Frente a lo expuesto, los objetivos de esta investigación fueron identificar el conocimiento de los individuos con DM tipo 102 registrados en el Programa HIPERDIA; correlacionar el tiempo de estudio con el número de respuestas concordantes acerca de los cuidados con los pies; y describir las complicaciones según el tiempo de diagnóstico.

\section{MATERIAL Y MÉTODOS}

Se trata de un estudio analítico, exploratorio y transversal, con análisis cuantitativo de los datos. La investigación fue desarrollada con individuos que presentan DM tipo 10 2 registrados y acompañados en el Programa HIPERDIA de una Unidad Matriz de Salud del municipio de Uberaba-MG.

Los individuos firmaron el Término de Consentimiento Libre y Aclarado y el proyecto de investigación fue aprobado por el Comité de Ética en Pesquisa de la Universidad Federal del Triângulo Mineiro - UFTM, bajo protocolo número 1829.

Se seleccionó a los individuos considerando los criterios de inclusión: personas con DM tipo 1 y 2, edad entre 19 a 59 años, concordaron en participar de la investigación y firmaron el Término de Consentimiento Libre y Aclarado. Los criterios de exclusión empleados fueron: individuos del sexo femenino con DM gestacional, que presentaban incapacidad mental/ intelectual para comprender y comunicarse (de acuerdo con informaciones recogidas en el prontuario), aquellos en cuyas casas fueron hechas más de tres visitas por los investigadores y no fueron encontrados en el domicilio, personas que cambiaron de dirección y los que no concordaron en participar de la investigación. 
En el período de junio a agosto de 2012 se realizó un levantamiento en los archivos del Programa HIPERDIA, siendo posteriormente seleccionados los que satisfacían los criterios de inclusión de este estudio, completando un total de 160 individuos registrados en la UMS.

De esa población, 15,6\% (25) cambiaron de dirección; 1,3\% (02) recusaron participar de la investigación; 0,6\% (01) no tuvieron condiciones de responder al cuestionario y $10 \%$ (16) no fueron encontrados en el domicilio después de tres visitas. Se tomaron entonces 116 individuos en este estudio. La recolección de datos fue realizada a partir de un cuestionario aplicado por los investigadores. Para evaluación y adecuación del instrumento, el mismo fue apreciado por dos especialistas en diabetes. Este instrumento se subdivide en tres partes: datos sociodemográficos (identificación, edad, sexo, procedencia, estado civil, escolaridad, situación productiva, profesión, religión y condiciones de vivienda), datos de salud (tipo de diabetes, tiempo de diagnóstico, medicación, complicaciones de la diabetes, Hipertensión Arterial sistémica (HAS), dislipidemia, orientaciones nutricionales, práctica de ejercicio físico, tabaquismo, etilismo, historia de amputaciones y lesiones previas) y datos referentes al cuidado con los pies. La construcción de este cuestionario fue realizada por las investigadoras, basada en el Consenso Internacional del Pie Diabético ${ }^{(9)}$. Además de eso, para confirmación de los datos de salud fue realizada consulta al archivo del individuo.

Para organización y análisis de los datos, estos fueron digitados en una planilla del programa Excel ${ }^{\circledR}$ en carácter de dupla entrada, para posterior validación y verificación de datos inconsistentes. El análisis estadístico fue realizado por medio del software del Statistical Package for the Social Sciences (SPSS) versión 17.0.

Para el primer objetivo se utilizó la estadística descriptiva por medio de frecuencias absolutas y porcentuales, medida de centralidad (media, mediana) y dispersión (desvío padrón, valores mínimos y máximo). En el segundo objetivo fue aplicado el test Correlación de Spearman. La intensidad de la correlación fue interpretada en muy baja $(r s=0 \mathrm{H} \mid 0,25)$, baja $(r s=0,26|-| 0,49)$, moderada $(r s=0,5-\mid 0,69)$, alta $(r s=$ $0,7+\mid 0,89)$ y muy alta $(r s=0,9+\mid 1,0)^{(10)}$. Para atender al tercer objetivo la variable tiempo de diagnóstico fue categorizada en: $<10$ años ó $\geq 10$ años y fue utilizado el test $x^{2}$.

El nivel de significancia $(\alpha)$ es de 0,05 y los test se consideraron significativos cuando $p<0,05$. Es necesario destacar que los valores de $p$ fueron interpretados suponiéndose que esta casuística constituye en una muestra aleatoria simple de una población con características similares.

\section{RESULTADOS Y DISCUSIÓN}

Los datos sociodemográficos de los individuos de ese estudio se encuentran presentes en la Tabla 1 . Se constató que $92,2 \%$ de los individuos tenían DM tipo 2, eran del sexo femenino $62,9 \%$, casada $65,5 \%$ y poseían enseñanza primaria incompleto $38,1 \%$, corroborando con los encontrados de un estudio realizado en una Unidad Básica de Salud, en el municipio de Ribeirão Preto (SP), en que $63,3 \%$ eran del sexo femenino y $63,3 \%$ casados $^{(11)}$. La media de edad fue de 49,9 años $(\mathrm{DP}=8,31)$, ese dato condice con otra investigación realizada en el interior de Minas Gerais ${ }^{(5)}$, en que la DM tipo 2 atacó más individuos $\geq 40$ años de edad. El hallazgo del presente estudio evidencia que $52,6 \%$ era natural de Uberaba. 
Tabla 1 - Distribución de los individuos con DM tipo 1 y 2, según las variables sociodemográficas. Uberaba - MG, 2012.

\section{Variables sociodemográficas}

n (\%)

\section{DM}

Tipo 1

$$
9(7,8)
$$

Tipo 2

$107(92,2)$

Sexo

Masculino

$43(37,1)$

Femenino

$73(62,9)$

\section{Estado civil}

Soltero

$16(13,8)$

Casado

$76(65,5)$

Viudo

Divorciado

$10(8,6)$

Unión Estable

\section{Escolaridad}

Analfabeto

Primaria incompleta

Primaria completa

Secundaria incompleta $11(9,5)$

Secundaria completa $34(29,3)$

Superior incompleta

Superior completa

$8(6,9)$

Sin respuestas

$3(2,6)$ 
Situación productiva

Empleo activo

$57(49,1)$

Jubilado

$26(22,4)$

Retirados con prestación

$10(8,6)$

Retirados sin prestación

$0(0,0)$

Desempleado

$23(19,8)$

Profesión

Empleado doméstico

$9(12,7)$

Comerciante

$5(7,0)$

Vendedor

$5(7,0)$

Otros

$97(73,3)$

\section{Con quien vive}

Padres

$6(5,2)$

Cónyuge

$17(14,7)$

Cónyuge e hijos

$42(36,2)$

Cónyuge, hijos y nietos

$13(11,2)$

Solo

$3(2,6)$

Otros

$35(30,1)$

Tipo de vivienda

Casa propia

$88(75,9)$

Casa alquilada

$18(15,5)$

Vivienda cedida

$9(7,7)$

Casa financiada

$1(0,9)$ 


\section{Tipo de suelo}

Cerámica

Cemento

Taco de madera

Cerámica/ taco

Cerámica/ taco/ cemento

Cerámica/ taco/ tierra

Cerámica/ cemento

\section{Tipo de Terreno}

Cimentado

Tierra / cimentado

Tierra

Ausente
$67(57,8)$

$11(9,5)$

$79(68,1)$

$21(18,1)$

$9(7,7)$

$3(2,6)$

$1(0,9)$

$1(0,9)$

$2(1,7)$

$2(1,7)$

En relación a la situación productiva hubo predominio de individuos con empleo activo. Mantenerse productivo y activo influye de forma positiva para una vida saludable con $\mathrm{DM}^{(12)}$. Se cree que individuos insertos en la convivencia social y en la actividad laboral, pueden presentar mayor interés y estímulo en tener más cuidado con la salud.

En cuanto a la vivienda, la mayoría de los individuos residían en casa propia $(75,9 \%)$ con piso de cerámica $(68,1 \%)$ y terreno cimentado $(57,8 \%)$. Conocer el tipo de piso de la residencia del diabético es de gran importancia para que el profesional de salud realice orientaciones a fin de prevenir accidentes en el domicilio y caídas, que pueden causar grandes traumas a los diabéticos que, muchas veces, poseen acentuada reducción visual ${ }^{(13)}$.

En la Tabla 2, está presentada la distribución de las variables relacionadas a los cuidados con los pies de los individuos diabéticos. 
Tabla 2 - Distribución de los cuidados con los pies realizados por los individuos con DM tipo 1 o 2 registrados en el HIPERDIA. Uberaba - MG, 2012.

\begin{tabular}{|c|c|c|}
\hline Variables & $\begin{array}{l}\text { Sí } \\
\text { n (\%) }\end{array}$ & $\begin{array}{l}\text { No } \\
\mathrm{n}(\%)\end{array}$ \\
\hline Cree importante cuidar de los pies & $114(98,3)$ & $2(1,7)$ \\
\hline Realiza examen de los pies con profesional de & & \\
\hline salud por lo menos una vez al año & $22(19,0)$ & $94(81,0)$ \\
\hline Realiza inspección diaria de los pies & $91(78,4 \%)$ & $25(21,6)$ \\
\hline Utiliza espejo para el autoexamen de los pies & $15(12,9)$ & $101(87,1)$ \\
\hline Higieniza diariamente los pies & $114(98,3)$ & $2(1,7)$ \\
\hline Realiza secado de los pies & $99(85,3)$ & $17(14,7)$ \\
\hline Acostumbra dejar los pies en agua & $56(48,3)$ & $65(51,7)$ \\
\hline Acostumbra usar zapatos apretados & $14(12,1)$ & $102(87,9)$ \\
\hline Acostumbra usar zapatos sin medias & $56(48,3)$ & $60(51,7)$ \\
\hline Acostumbra cambiar de zapatos diariamente & $106(91,4)$ & $10(8,6)$ \\
\hline Acostumbra usar medias con costuras & $85(73,3)$ & $31(26,7)$ \\
\hline Acostumbra caminar descalzo & $34(29,3)$ & $82(70,7)$ \\
\hline Inspecciona los zapatos antes de usar & $78(67,2)$ & $38(32,8)$ \\
\hline $\begin{array}{l}\text { Notifica al equipo de salud si tuviera lesiones } \\
\text { en los pies }\end{array}$ & $68(58,6)$ & $48(41,4)$ \\
\hline Elimina callos con bisturí, entre otros & $33(28,4)$ & $83(71,6)$ \\
\hline $\begin{array}{l}\text { Corta uñas de forma recta sin limar los } \\
\text { bordes }\end{array}$ & $77(66,4)$ & $39(33,6)$ \\
\hline $\begin{array}{l}\text { Acostumbra quitar cutículas de las uñas } \\
\text { Pide ayuda para algún familiar cuando no }\end{array}$ & $66(56,9)$ & $50(43,1)$ \\
\hline consigue realizar el cuidado solo & $81(69,8)$ & $35(30,2)$ \\
\hline $\begin{array}{l}\text { Considera el apoyo familiar importante en el } \\
\text { cuidado con los pies }\end{array}$ & $111(95,7)$ & $5(4,3)$ \\
\hline
\end{tabular}

En relación a los cuidados con los pies, 98,3\% de los individuos consideraba importante cuidar de los pies para prevenir el pie diabético, mientras, $81 \%$ dijeron no realizar examen de los pies por lo menos una vez al año con el profesional de salud. La mayor parte de los individuos del presente estudio mantenían conductas adecuadas en su día a día para el cuidado de los pies. Entre ellas: higienizar $(98,3 \%)$, inspeccionar $(78,4 \%)$ y realizar el secado diariamente $(85,3 \%)$, no acostumbra dejar los pies en agua (51,7\%), no caminar descalzo (70,7\%), no usar zapatos apretados $(87,9 \%)$, cortar uñas de forma recta sin limar los bordes $(66,4 \%)$, no acostumbra usar zapatos sin medias $(51,7 \%)$, realizar inspección de los zapatos antes de usar $(67,2 \%)$, cambiar los zapatos diariamente $(91,4 \%)$, pedir ayuda a algún familiar cuando no consigue realizar el cuidado solo $(69,8 \%)$ y notificar al equipo de salud si tuviese alguna lesión en los pies (58,6\%) (Tabla 2).

Entre los individuos que dijeron secar los pies, 88,8\% mencionaron que realizaban el secado de la planta, dorso de los pies y entre los dedos, mientras que $11,2 \%$ secaban la planta y dorso de los pies. El hábito de no secar los pies entre los dedos, puede propiciar la proliferación de micro-organismos y ocasionar fisuras en la piel.

En relación a la hidratación de los pies 68,1\%, dijeron que acostumbraban hidratar, siendo que $37,1 \%$ lo hacían en la planta y dorso solo, 31,0\% hidrataban planta, dorso y entre los dedos, mientras que $31,9 \%$ no hidrataban. Para evitar el resecamiento de 
la piel y así, prevenir que aparezcan lesiones, se deben mantener hidratadas las piernas y pies ${ }^{(14)}$.

En cuanto a las prácticas inadecuadas para los cuidados de los pies, $73,3 \%$ de los individuos acostumbraban usar medias con costuras y $56,9 \%$ remover cutículas de las uñas. Se resalta que esas prácticas pueden ofrecer riesgo a la integridad de la piel de los pies, principalmente para aquellos que poseen neuropatía, visto que estos individuos poseen sensibilidad disminuida.

Las orientaciones con los cuidados para la prevención del pie diabético consisten en una herramienta imprescindible, y esta debe ser una práctica constante en la rutina de los profesionales de salud, en especial del enfermero.

En este estudio hubo predominio de las respuestas de los diabéticos que consideraban el apoyo familiar importante en el cuidado con los pies (95,7\%). Las relaciones familiares consisten en significante factor para la adhesión al tratamiento en el inicio de $\mathrm{DM}^{(15)}$.

Los datos referentes a hábitos y variables de salud se presentan a continuación:

Tabla 3 - Distribución de los individuos que presentan DM según los hábitos y variables de salud. Uberaba - MG, 2012.

\begin{tabular}{lcc}
\multicolumn{1}{c}{ Hábitos e Variables } & $\mathbf{S i}$ & $\mathbf{N o}$ \\
$\mathbf{n}$ & $\mathbf{n}(\%)$ & $36(31,0)$ \\
Dislipidemia & $80(69,0)$ & $63(54,3)$ \\
Tabaquismo & $53(45,7)$ & $95(81,9)$ \\
Etilismo & $21(18,1)$ & $106(91,4)$ \\
Orientaciones & $10(8,6)$ & $43(37,1)$ \\
nutricionales & $73(62,9)$ & \\
Ejercicio físico & $50(43,1)$ & $66(56,9)$ \\
\hline
\end{tabular}

En relación al tiempo de diagnóstico de la DM, la media fue de 5 años (Min=0,6; Máx=32). La mayoría de los individuos presentaban HAS asociada a DM (Tabla 3), y tiempo diagnóstico con media de 9 años (Min=0,5; Máx=30). Otros factores de riesgo fueron observados en los individuos del presente estudio, entre ellos la dislipidemia media del tiempo diagnóstico 4,5 años (Min=0,1; Máx.=20), o tabaquismo media del tiempo diagnóstico 30 años (Mín.=4; Máx.=50), el etilismo media del tiempo diagnóstico 20 años (Min=3; Máx.=40). Otros estudios ${ }^{(7,16)}$ también refieren resultados semejantes, se encontró que $66,0 \%$ presentaba hipertensión arterial y $49 \%$ dislipidemia.

En cuanto a los individuos que dijeron practicar ejercicio físico (Tabla 3), 56,0\% citaron caminar, $14,0 \%$ hacer gimnasio, 10,0\% pedalear, 6,0\% hidro-gimnasia y $16,0 \%$ realizaban esas actividades asociadas. Con base en estos datos se comprobó semejanza con otros hallazgos ${ }^{(13)}$ que la actividad física fue referida solamente por $40,6 \%$ de los entrevistados y se destacó la caminata. La mayor parte de la población del presente estudio (62,9\%) dijo seguir las orientaciones nutricionales.

Estudios realizados demostraron que hábitos de vida más saludables (dieta balanceada asociada a la actividad física regular) pueden disminuir en $58 \%$, el riesgo de que individuos pre-diabéticos desarrollen $\mathrm{DM}^{(17)}$. En el caso de individuos con DM 
ya instalada, esos hábitos contribuyen de forma significativa en el control de la enfermedad y en la prevención de complicaciones graves.

De acuerdo con el tipo de tratamiento, se comprobó que 63,8\% hacían uso de hipoglucemiante oral, $12,0 \%$ usaban apenas insulina, 17,2\% hipoglucemiante oral asociado a la insulina e $8,6 \%$ realizaban tratamiento no medicamentoso. Otro estudio $^{(13)}$ realizado en una Estrategia de Salud de la Familia en una ciudad de Minas Gerais encontró que 66,6\% utilizaban hipoglucemiantes orales, $7,7 \%$ solamente de insulina, $7,7 \%$ hipoglucemiantes orales junto con la insulina y $17,9 \%$ realizaban el control de la diabetes apenas con dieta.

Se puede destacar que $58,3 \%$ de los individuos del referido estudio nunca tuvieron lesión en los pies y 41,7\% dijeron tener o tuvieron lesión en los pies. Entre los que afirmaron 10,3\% trataron la lesión en el hospital, 8,6\% en la Unidad de Salud, 21,6\% en casa, y $0,9 \%$ no se trató. Se cree que los profesionales de salud, en especial el enfermero, deban estar atentos y acompañar con mayor frecuencia a estos individuos, principalmente a los que se tratan en casa para evaluar la lesión evitando así complicaciones

En cuanto a la forma de tratamiento de la lesión, 25,0\% de los individuos utilizaban pomada, $4,3 \%$ usaban antiséptico, $1,7 \%$ se hacían baño de hierbas, $2,6 \%$ respondieron que trataban de otras formas (antibiótico y cirugía), 2,6\% pomadas y antiséptico, 1,7\% pomadas y otros, siendo que apenas $0,9 \%$ fue para amputación.

El uso de pomada para el tratamiento de las lesiones corrobora con otro estudio en el cual el cuidado mayor se dio por el uso de pomadas y remedios para la terapéutica ${ }^{(18)}$.

El desarrollo de habilidades para el cuidado de los pies es una condición impar para la educación de los diabéticos ${ }^{(19)}$.

La media de las respuestas concordantes fue de 13,53, en lo que se refiere a la media de las respuestas discordantes el valor fue 7. Los tests estadísticos mostraron que hubo correlación baja entre el tiempo de estudio y el número de respuestas concordantes acerca de los cuidados de los pies, pero no fue significativa $(r s=0,026$; $p=0,781)$.

El resultado obtenido no corrobora con otros estudios, en los cuales se demuestra que personas con edad avanzada y baja escolaridad pueden presentar una dificultad mayor para comprensión y adhesión al tratamiento propuesto para control de la diabetes y sus complicaciones ${ }^{(20,21)}$.

Entre aquellos con hasta 10 años de diagnóstico, 79,7\% no dijeron presentar complicaciones, mientras $62,2 \%$ para aquellos con 10 años o más mencionó tener complicaciones $\left(x^{2}=19,83 ; p<0,001\right)$.

De acuerdo con investigadores cuanto mayor es el tiempo de diagnóstico, mayor la proporción de individuos con complicaciones crónicas, pues la precocidad del diagnóstico, juntamente con la adhesión al tratamiento es relevante en la prevención de gravedad ${ }^{(5)}$. Cuanto mayor es el tiempo al diagnóstico, menor a prevalencia de la adhesión al tratamiento aumentando el riesgo de complicaciones resultantes del control metabólico insatisfactorio ${ }^{(22)}$. 
Se destaca entre los individuos que presentaban 10 años o más de tiempo de diagnóstico de DM que la mayoría $(56,5 \%)$ presentó complicaciones microvasculares, $17,4 \%$ complicaciones macrovasculares, y $26,1 \%$ tenían tanto complicaciones micro vasculares como macrovasculares. Para los individuos con hasta 10 años de diagnóstico, hubo prevalencia $(56,2 \%)$ en las complicaciones microvasculares, seguido de $31,2 \%$ que tuvieron complicaciones macrovasculares, mientras que $12,5 \%$ de los individuos tenían complicaciones micro y macrovasculares. Lo que consiste en un factor agravante, pues, si antes de alcanzar 10 años de diagnóstico la mayoría de esa población ya presenta complicaciones, se puede deducir que estos van a presentar incapacidades físicas.

Las complicaciones derivadas de la DM acarrean incapacidad para el cumplimiento de actividades de rutina y disminución de la calidad de vida ${ }^{(23)}$.

Otro estudio evidenció que es posible la prevención de las complicaciones derivadas de la DM. Esto habla de la necesidad de reforzar, junto a los servicios de salud en la esfera municipal, medidas y acciones preconizadas por el Ministerio de Salud, buscando disminuir los factores de riesgo y el impacto de esas complicaciones en la calidad de vida de los diabéticos Tipo 2, para que los mismos consigan ser más saludables y participativos en la sociedad ${ }^{(24)}$.

La prevención debe ser considerada como el mayor foco en el abordaje del pie diabético y así en la reducción de las amputaciones. Para obtener mayor adhesión a los cuidados necesarios es importante el diagnóstico precoz, la sensibilización y orientación del individuo y de su familia en cuanto a esa complicación. Algunos de los cuidados incluyen rutinas de higiene diaria, restricciones a caminar descalzo y orientaciones sobre calzados adecuados ${ }^{(4)}$.

Se verifica la necesidad de estimular a los individuos para la incorporación de prácticas adecuadas de control de la DM, sobre todo el cuidado con los pies, mirando nuevos conocimientos y sensibilización en cuanto a la forma de lidiar con la enfermedad. El enfermero en la consulta de enfermería posee contacto estrecho con esa población, presentando papel fundamental en esas acciones, proporcionando mecanismos que pueden ser utilizados para identificar, acompañar y realizar intervenciones.

La consulta de enfermería configura una importante manera de seguir la pista de los pacientes con riesgo para desarrolar úlceras de miembros inferiores ${ }^{(3)}$.

Los enfermeros poseen papel importante en la implementación de acciones educativas que permiten el aumento del conocimiento de la población en relación a la prevención de complicaciones de la $\mathrm{DM}^{(16)}$.

\section{CONCLUSIÓN}

El presente estudio demostró que los individuos diabéticos tenían cierto conocimiento sobre la DM y entendieron la importancia de la inspección diaria y del cuidado con los pies para prevenir el pie diabético. En su mayoría tenían conductas adecuadas en el cotidiano en relación al cuidado de los pies, sin embargo, todavía existen individuos que presentan complicaciones lo que hace imprescindible la realización de medidas de prevención y el acompañamiento por los profesionales de salud, principalmente del enfermero. 
Tener conciencia del nivel de conocimiento del individuo diabético sobre los cuidados de los pies permite dirigir las acciones de educación en salud que lleven a promover e incentivar esta práctica.

Se sabe que el tiempo de estudio puede interferir en el conocimiento de los individuos en relación al cuidado con la salud, mientras, en el presente estudio no fue un factor limitante el número de respuestas concordantes acerca de los cuidados con los pies. En relación a las complicaciones derivadas de la DM, se notó que estas son más frecuentes en individuos con 10 años o más de tiempo de diagnóstico.

Este estudio fue de gran valor para la población, pues demuestra la importancia de identificar los factores de riesgo para el desarrollo de complicaciones, y también conocer el contexto en que el individuo está inserto. Esas cuestiones podrían ser mejor identificadas por el enfermero durante la consulta de enfermería permitiendo mejora de las acciones, así como, una intervención sistematizada por medio de un acompañamiento directo y educación en salud.

\section{REFERENCIAS}

1. Sociedade Brasileira de Diabetes. Diretrizes da Sociedade Brasileira de Diabetes. 3 ed. São Paulo: Itapevi, 2009.

2. Brasil. Ministério da saúde. Vigilância de fatores de risco e proteção para doenças crônicas por inquérito telefônico (VIGITELBrasil 2011). Dados sobre diabetes. [Acesso em 2013, jan, 29]. Disponível em: $<$ http://portalsaude.saude.gov.br/portalsaude/arquivos/pdf/2012/Mai/09/Vigitel_2011_d iabetes_final.pdf>.

3. Brasil. Ministério da Saúde. Secretaria de Atenção à Saúde. Departamento de atenção Básica. Cadernos de Atenção Básica no 16: Diabetes Mellitus, Brasília (DF): Ministério da Saúde, 2006.

4. Parisi, C. A síndrome do pé diabético e aspectos práticos e fisiopatologia. Sociedade Brasileira de Diabetes. Diabetes na prática clínica. Cap. 5, 2011.

5. Carvalho, R.D.P; Carvalho, C.D.P; Martins, D.A. Aplicação dos cuidados com os pés entre portadores de Diabetes mellitus. CogitareEnferm. 2010 Jan/Mar; 15(1):106109.

6. Rocha, R.M; Zanetti, M.L; Santos, M.A. Comportamento e conhecimento: fundamentos para prevenção do pé diabético. Acta Paul Enferm. 2009; 22(1):17-23.

7. Laurindo, M.C; Recco, D.C; Robert, D.B; Rodrigues, C.D.S. Conhecimento das pessoas diabéticas acerca dos cuidados com os pés. Arq Ciên Saúde. 2005; 12(2): 80-4.

8. Gil, G.P; Haddad, M.C.L; GuarientE, M.H.D.M. Conhecimento sobre diabetes mellitus de pacientes atendidos em programa ambulatorial interdisciplinar de um hospital universitário público. Semina cienc biol saúde. Jul/dez 2008; 29 (2): 141-54.

9. Grupo de trabalho sobre pé diabético. Consenso internacional sobre o pé diabético. Brasília: Secretaria de Estado de Saúde do Distrito Federal, 2001.

10. Munro, B.H. Statistical methods for health care researsh. Philadelphia: Lippincott, 2001. $459 \mathrm{p}$.

11. Oliveira, K.C.S; Zanetti, M.L. Conhecimento e atitude de usuários com diabetes mellitus em um serviço de atenção básica à saúde. Rev. esc. enferm. USP. 2011 [acesso em 30 jul 2012]. Disponível em: <http://dx.doi.org/10.1590/S0080$62342011000400010>$. 
12. Francioni, F.F; Silva, D.G.V. O processo de viver saudável de indivíduos com Diabetes Mellitus através de um grupo de convivência. Texto contexto - enferm. 2007; 16 (1): 105-111.

13. Barbui, E.C; Cocco, M.I.M. Conhecimento do cliente diabético em relação aos cuidados com os pés. Rev. Esc Enferm USP. 2002; 36 (1): 97-103.

14. Amaral, A.S Tavares, D.M.S. Cuidados com os pés: conhecimento entre indivíduos com diabetes mellitus. Rev. Eletr. Enf. 2009;11(4): 801-10.

15. Malerbi, F.E.K. Adesão ao tratamento, importância da família e intervenções comportamentais em diabetes. Sociedade Brasileira de Diabetes. Diabetes prática clínica. Cap. 7, 2011.

16. Gack Ghelman, L.; Souza, MH Do N.; Machado TInoco Feitosa Rosas, AM Conhecimento de portadores de Diabetes Mellitus atendidos em uma Unidade Básica de Saúde, quanto às práticas de auto-cuidado com pés. Enfermería Global. ํํ 17, Octubre 2009.

17. Ferreira, S.R.G. Aspectos epidemiológicos do diabetes mellitus e seu impacto no indivíduo e na sociedade. Sociedade Brasileira de Diabetes. Diabetes na prática clínica. Cap. 1, 2011.

18. Coelho, M.S; Silva, D.M.G.V; Padilha, M.I.S. Representações sociais do pé diabético para indivíduos com diabetes mellitus tipo 2. Rev. esc. enferm. USP . 2009,43(1): 65-71.

19. Martin IS, Beraldo AA, Passeri SM, Freitas MCF, Pace AE. Causas referidas para o desenvolvimento de úlceras em pés de indivíduos com diabetes mellitus. Acta paul. enferm. 2012, 25 (2): 218-224.

20, Assunção, T.S; Ursine, P.G.S. Estudo de fatores associados à adesão ao tratamento não farmacológico em portadores de Diabetes Mellitus assistidos pelo Programa Saúde da Família, Ventosa, Belo Horizonte. Cien Saude Colet. 2008; 13 (Supl2): 2189-97.

21. Assunção, M.C.F.; Santos, I.S.; Costa, J.S. Avaliação do processo da atenção médica: adequação de pacientes com diabetes mellitus. Cad Saúde Pública 2002;18(1):205-211

22. Rodrigues, F.F.L; Santos, M.A; Teixeira, C.R.S.; Gonela, J.T.; Zanetti, M.L. Relationship between knowledge, attitude, education and duration of disease in individuals with diabetes mellitus. Acta Paul Enferm. 2012;25(2):284-90.

23. Oliveira, G.K.S; Oliveira, E.R. Assistência de enfermagem ao portador de diabetes mellitus: um enfoque na atenção primária em saúde. Revista Eletrônica de Ciências 3 (2) julho a dezembro de 2010.

24. Santos, I.C.R.V; Carvalho, E.F; Souza, W.V; Medeiros, M.C.W.C.; Nóbrega, M.G.L.; LIma, P.M.S. Complicações crônicas dos diabéticos tipo 2 atendidos nas Unidades de Saúde da Família, Recife, Pernambuco, Brasil. Rev. Bras. Saude Mater. Infant. 2008 [acesso em 20 dez 2012]. Disponível em: <http://dx.doi.org/10.1590/S1519-38292008000400008>.

Recibido: 7 de marzo de 2013; Aceptado: 27 de abril de 2013

ISSN 1695-6141

๑) COPYRIGHT Servicio de Publicaciones - Universidad de Murcia 